\title{
A PTDOA-DRSS Hybrid Factor Graph-based Unknown Radio Wave Geolocation
}

\author{
Shofiyati Nur Karimah ${ }^{1}$, Muhammad Reza Kahar Aziz ${ }^{2}$, and Tad Matsumoto ${ }^{1,3}$ \\ ${ }^{1}$ Japan Advanced Institute of Science and Technology (JAIST), 1-1 Asahidai, Nomi, Ishikawa, 923-1292 JAPAN \\ ${ }^{2}$ Electrical Engineering, Institut Teknologi Sumatera (ITERA), Lampung Selatan, 35365 INDONESIA \\ ${ }^{3}$ Centre for Wireless Communications, University of Oulu, Oulu, 90014 FINLAND \\ Email: \{karimah,matumoto@jaist.ac.jp\},reza.kahar@el.itera.ac.id
}

\begin{abstract}
We propose a hybrid Pythagorean Time Difference of Arrival and Differential Received Signal Strength based Factor Graph (PTDOA-DRSS-FG) to estimate the location of unknown radio wave emitter in outdoor environments. The term of "unknown" indicates that the knowledge of neither time of departure (TOD) nor absolute transmit power of the radio wave emitter are required. The PTDOA-FG can eliminate the necessity of TOD knowledge of the target signal transmission and DRSS-FG can eliminate the necessity of the knowledge of target absolute transmit power. However, PTDOA-FG alone requires perfect time synchronism between sensors, which is difficult in practice. On the other hand, DRSS-FG alone requires the most suitable monitoring spots. In this paper, PTDOA-FG is used to provide the rough estimation of the target position to select the most suitable monitoring spots for DRSS-FG technique. It is shown that, in terms of root mean square error (RMSE) vs. iteration times, the achieved RMSE of the proposed technique is better than the PTDOA-FG alone and very close to the DRSS reference, i.e., the DRSS-FG technique with the idealistic monitoring spots. Performing the proposed technique in the framework of factor graph (FG) does not require excessive computational complexity due to the fact that using the Gaussian distribution model, it uses mean and variance only with the sum-product algorithm.
\end{abstract}

\section{Introduction}

Geolocation is a multidisciplinary topic that has been recognized as a key to provide location-based services in recent and future mobile communication systems, e.g., smart transportation systems, fraud detection, missing object detection, wireless security, and public safety systems. The current trend of Geolocation is to establish low-cost, high accuracy, and low complexity techniques. Furthermore, another challenging problem in geolocation is that estimating the location of unknown target, where the term "unknown" in this paper indicates that the knowledge of neither time of arrival (TOA) nor transmit power of the radio wave emitter, are required [1], [2], [3].

Wireless geolocation is defined as the process to calculate a geographical coordinate of radio wave emitter by observing the electromagnetic wave properties [1]. The measurement of electromagnetic wave properties such as TOA, received signal strength (RSS), time difference of arrival (TDOA), and their combinations are utilized to estimate the geographical location of a radio wave emitter.

We present techniques that performed geolocation through combining the measurements of RSS and TOA in [4] as well as combining the RSS and direction of arrival (DOA) in [5]. The results of joint techniques show better accuracy than the utilization of each parameter alone, i.e., RSS, TOA, and DOA. However, to obtain DOA information, antenna arrays and/or directional-antenna are needed, which impose significant hardware complexity. Even though hybrid RSS-TOA technique is less costly due to the unnecessity of additional hardware, it cannot be applied to estimate the position of unknown radio wave emitter since it is almost impossible to extract the absolute transmit power and the time of departure (TOD) information from the unknown target.

To estimate the position of unknown wave emitter, we propose in this paper a hybrid technique by combining Pythagorean time difference of arrival (PTDOA), and differential signal strength (DRSS) based Factor Graph, where each of PTDOA-alone and DRSS-alone technique has been presented in [1], [6]. In the same way in [4], the hybrid use of RSS and TOA as [4] can achieve higher accuracy while keeping computational complexity low.

The use of factor graph (FG) in the proposed hybrid technique Pythagorean Time Difference of Arrival and Differential Received Signal Strength based Factor Graph (PTDOA-DRSS-FG) makes it easy to describe the algorithm structure, where the global function is separated into several local functions [7]. Fig. 1 shows the entire structure of the proposed technique in a single FG. There are two nodes in the FG, i.e., (1) the square shape is called factor node, which calculates the updated Soft Information (SI) messages; representing mean and variance of parameters required to convert the location of the target position at the final stage; (2) the round shape is called the variable node, which performs the sum-product algorithm and the values of the node output are forwarded to the factor nodes. It should be noticed that only means and variances of measurement data are exchanged between the factor and variable nodes because of the Gaussianity assumption of the measurement data.

\subsection{Related Work}

Ref. [8] first introduces an FG based geolocation technique which performs the sum-product algorithm 


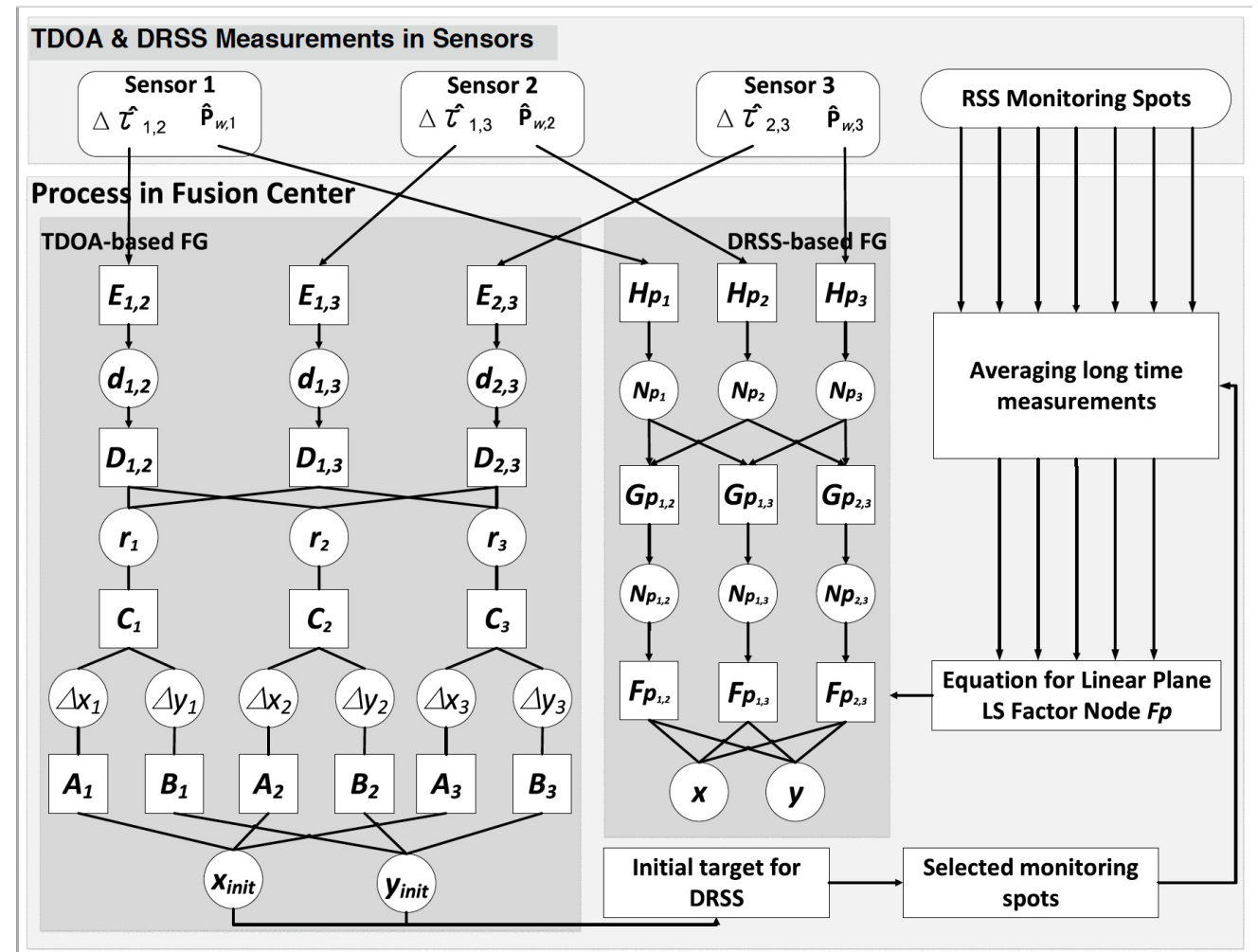

Figure 1: The proposed PTDOA-DRSS-based FG.

to estimate the location of a target using TOA. Only mean and variance of TOA measurement, assumed to be Gaussian-distributed, are exchanged between the nodes. Each sensor performs multiple measurements of TOA and calculates the mean and variance empirically.

Ref. [9] proposes a TOA-based FG technique which is basically the same as the technique shown in [10]. However, it takes into account that the measurement error of TOA is included in measured data of each sensor, in the process of the conversion of the parameters to the distance. Nevertheless, direct use of TOA is not practical because it requires perfect time synchronisation of the target which is impossible if the target is unknown. Therefore, to eliminate the necessity of the time stamp knowledge, we have proposed Pythagorean TDOA-based FG (PTDOA-FG) in [1] by modifying the TOA-based FG technique in [9].

Ref. [11] proposes a geolocation technique using RSS-based FG. It requires knowledge of RSS obtained by measuring the strength of the received training signal, transmitted from monitoring spots. The obtained training reference of RSS data in the unit of watt is converted to $\mathrm{dB}$ as the input of the FG. The RSS reference obtained through the training is then used to produce path-loss profile by interpolation, where local linearity is exploited to estimate the target location. In an indoor scenario with the size of $100 \mathrm{~m}^{2}$, Ref. [11] shows that the technique can achieve accuracy of $0.6-0.9 \mathrm{~m}$ with the standard deviation (STD) of the measurement data $2 \times 10^{-6}$ $7 \times 10^{-6}$ watts.

To eliminate the necessity of absolute transmit power knowledge of unknown radio wave emitter, we also have proposed DRSS-based FG (DRSS-FG) in [1] and [6] by modifying RSS-based FG technique in [11], which works in the differential domain of RSS parameter between two sensors. Hence, the subtraction of RSS is required as a new node in the FG [11]. However, due to the use of least square formula, as mentioned in [11], the most suitable monitoring spots is in square formation. Furthermore, their positions have to be the closest to, and surrounding the target. The DRSS-FG in [1] assumes the most suitable monitoring spots are always known, i.e., the target is located in the middle of the monitoring spot grid. In practice, there are many monitoring spots available, hence, finding them requires a help by another algorithm. To identify the appropriate monitoring spot, Ref. [11] uses RADAR algorithm in [12], whilst in Ref. [13], we utilize Voronoi diagram for RSS-based FG in an outdoor environment. However, both RADAR algorithm and Voronoi diagram require the knowledge of transmit power of the target.

Therefore, to identify the suitable initial point, this paper proposes the use of PTDOA-FG, which selects the monitoring spots for DRSS-FG in outdoor environments. The mechanism of combining two schemes is based on the scheme proposed in [4], i.e., using the output of one existing method, PTDOA-FG, as the input of the other existing method, DRSS-FG.

\subsection{Contributions}

This paper's major contributions are (1) to propose an algorithm of hybrid PTDOA and DRSS-FG, with the aim of its application to outdoor environments, and (2) to investigate its accuracy through computer simulations and then compare the estimation utilizing PTDOA-FG 
alone. The hybrid PTDOA-DRSS-based FG algorithm, as shown in Fig. 1, utilizes parameters of differential TOA and RSS which are obtained by the subtraction between two sensors. The result of the PTDOA-FG estimation algorithm at the variable nodes $\left(x_{i n i t}, y_{i n i t}\right)$, then is used as the initial point for DRSS-FG as well as becomes a rough estimate of the target position for selecting the four most appropriate monitoring spots. The final variable nodes of DRSS-FG $(x, y)$ is the final estimate of the target position. In this paper, for the accuracy comparison, we also evaluate the case where (1) the initial point position for estimating the actual target position is idealistic, e.g., the case where the initial point is in the same grid of monitoring spots as the real target position, and (2) the case where the initial point is not necessarily be in the same grid of the monitoring spot as the target position requires.

\section{System Model of Hybrid PTDOA-DRSS-FG Technique}

The system consists of one target $(x, y)$, multiple $N$ sensors $\left(X_{h}, Y_{h}\right)$, where $h$ is indicating the sensor index, $h=1,2, \cdots, N$, and several monitoring spots $\left(x_{m}, y_{m}\right)$, where $m$ indicates the monitoring spots index. Both PTDOA-FG and DRSS-FG have nodes performing message subtraction between any sensor pairs, $i$ and $j$ which $i \neq j$ and $\{i, j\} \in h$.

The target is a static unknown (anonymous) radio wave emitter. While, the positions of sensors as well as the monitoring spots are known to the fusion center. Each sensor sends the information of TDOA, i.e., the different TOA between two sensors, $i$ and $j, \Delta \hat{\tau}_{i, j}$, that is furthermore converted to the distance $\hat{d}_{i, j}$ taking into account the velocity of light, and received signal power at the sensors, $i$ and $j$, in the unit of watt, $\hat{\mathcal{P}}_{w_{i}}$ and $\hat{\mathcal{P}}_{w_{j}}$, to the fusion center. The RSS samples at each monitoring spot are obtained through the offline training process.

The RSS samples, including the training data and that of the target, are assumed to be expressed by path-loss exponent model, of which this assumption hold, averaging over long enough duration of measurement is needed to eliminate the instantaneous fading variation and shadowing. Due to the variation accumulated effects of many independent factors, the measurement error can be modeled as non-zero Gaussian noise [9], [11]. However, the process of obtaining measurement data is out of scope in this paper.

In this section, we briefly explain the PTDOA and DRSS FG algorithms introduced in [1] independently, as the initial step towards developing the proposed technique.

\subsection{PTDOA-FG Technique}

The Pythagorean scheme used in PTDOA-FG is a modification of Pythagorean scheme presented in [9]. In [1], we locate several additional set of nodes to eliminate the necessity of time stamp knowledge of the target signal transmission. Furthermore, instead of TOA, the input to the FG is TDOA between sensor pairs.

For smooth understanding, we describe the PTDOA-FG step by step as follow.
Step-1 TOA denotes the time duration between the signal departure at the transmitter and arrival at the receiver. In this case, the transmitter is the target and the receiver is the sensor. TOA $\hat{\tau}$ can be expressed as

$$
\hat{\tau}=\hat{\tau}_{a}-\hat{\tau}_{d}
$$

where $\hat{\tau}_{a}$ is the time when signal arrives at the sensor, and $\hat{\tau}_{d}$ is time when the signal departs from the target. Note that for the simplicity, we omit the sensor index in (1). For the unknown target, the information of departure time $\hat{\tau}_{d}$ is not known. Hence, [1] utilizes TDOA $\Delta \hat{\tau}$ to eliminate the necessity of $\hat{\tau}_{d}$ knowledge by subtracting $\hat{\tau}_{a}$ between sensors, as

$$
\begin{aligned}
\Delta \hat{\tau}_{i, j} & =\hat{\tau}_{i}-\hat{\tau}_{j} \\
& =\left(\hat{\tau}_{a_{i}}-\hat{\tau}_{d}\right)-\left(\hat{\tau}_{a_{j}}-\hat{\tau}_{d}\right) \\
& =\hat{\tau}_{a_{i}}-\hat{\tau}_{a_{j}} .
\end{aligned}
$$

The step for obtaining TDOA $\Delta \hat{\tau}_{i, j}$ applies to all the sensors, as shown in Fig. 1.

Step-2 In the fusion center, the TDOA sample can be directly converted to the difference of Euclidean distance, $\hat{d}_{i, j}=\Delta \hat{\tau}_{i, j} \cdot c$, where $c$ is the velocity of electromagnetic wave propagation $3 \times 10^{8} \mathrm{~m} / \mathrm{s}$. Referring to the FG shown in Fig. 1, factor node $E_{i, j}$ converts $K$ samples into difference of Euclidean distance and then calculates the mean $m_{E_{i, j} \rightarrow d_{i, j}}$ and variance $\sigma_{E_{i, j} \rightarrow d_{i, j}}^{2}$ as the message to be exchanged in the FG. The subscript arrow $\rightarrow$ indicates the flow of messages among the nodes in the FG.

Step-3 Since there are only two nodes directly connected to variable node $d_{i, j}$, it passes the message from the factor node $E_{i, j}$ to the factor node $D_{i, j}$ in the form of mean $m_{d_{i, j} \rightarrow D_{i, j}}$ and variance $\sigma_{d_{i, j} \rightarrow D_{i, j}}^{2}$ as

$$
\begin{gathered}
m_{d_{i, j} \rightarrow D_{i, j}}=m_{E_{i, j} \rightarrow d_{i, j}}, \\
\sigma_{d_{i, j} \rightarrow D_{i, j}}^{2}=\sigma_{E_{i, j} \rightarrow d_{i, j}}^{2} .
\end{gathered}
$$

Step-4 Factor node $D_{i, j}$, so called Euclidean distance estimator node, converts the difference Euclidean distance to the equivalent Euclidean distance, given by the following operations:

$$
\begin{gathered}
m_{D_{i, j} \rightarrow r_{i}}=m_{d_{i, j} \rightarrow D_{i, j}}+m_{r_{j} \rightarrow D_{i, j}}, \\
m_{D_{i, j} \rightarrow r_{j}}=-m_{d_{i, j} \rightarrow D_{i, j}}+m_{r_{i} \rightarrow D_{i, j}}, \\
\sigma_{D_{i, j} \rightarrow r_{i}}^{2}=\sigma_{d_{i, j} \rightarrow D_{i, j}}^{2}+\sigma_{r_{j} \rightarrow D_{i, j}}^{2}, \\
\sigma_{D_{i, j} \rightarrow r_{j}}^{2}=\sigma_{d_{i, j} \rightarrow D_{i, j}}^{2}+\sigma_{r_{i} \rightarrow D_{i, j}}^{2} .
\end{gathered}
$$

Step-5 Variable node $r_{h}$, with subscript $h$ means the sensor index, $\{i, j\} \in h$, updates the messages from the node $D_{i, j}$ and $C_{h}$ according to the sum-product algorithm, and then forwards the updated messages to the destination nodes. The updated messages from the variable node $r_{h}$ to the factor node $D_{i, j}$ are expressed in (9) and (10), whilst (11) and (12) express the updated messages from the variable node $r_{h}$ to the factor node $C_{h}$. The mean $m_{C_{h} \rightarrow r_{h}}$ and variance $\sigma_{C_{h} \rightarrow r_{h}}^{2}$ indicate the messages from factor node $C_{h}$, as 


$$
\begin{aligned}
& \frac{1}{\sigma_{r_{h} \rightarrow D_{i, j}}^{2}}=\left(\sum_{n=1, n \neq i, n \neq j}^{N} \frac{1}{\sigma_{a}^{2}}\right)+\frac{1}{\sigma_{C_{h} \rightarrow r_{h}}^{2}}, \\
& m_{r_{h} \rightarrow D_{i, j}}=\sigma_{r_{h} \rightarrow D_{i, j}}^{2} \cdot \\
&\left(\left(\sum_{n=1, n \neq i, n \neq j}^{N} \frac{m_{a}}{\sigma_{a}^{2}}\right)+\frac{m_{C_{h} \rightarrow r_{h}}}{\sigma_{C_{h} \rightarrow r_{h}}^{2}}\right), \\
& \frac{1}{\sigma_{r_{h} \rightarrow C_{h}}^{2}}=\sum_{n=1, n \neq h}^{N} \frac{1}{\sigma_{a}^{2}}, \\
& m_{r_{h} \rightarrow C_{h}}=\sigma_{r_{h} \rightarrow C_{h}}^{2} \cdot\left(\sum_{n=1, n \neq h}^{N} \frac{m_{a}}{\sigma_{a}^{2}}\right),
\end{aligned}
$$
$h>n$.

where $a=D_{h, n} \rightarrow r_{h}$ for $h<n, a=D_{n, h} \rightarrow r_{h}$ for

The messages exchanged between node $r_{h}$ and $D_{i, j}$ eliminate the necessity of the time stamp knowledge, and hence plays crucial role in the algorithm. Then, in the first iteration, we set the initial value of mean $m_{C_{h} \rightarrow r_{h}}=0$ and variance $\sigma_{C_{h} \rightarrow r_{h}}^{2}=1$.

Step-6 The Pythagorean node $C_{h}$ is the critical node because it performs Pythagorean operations to update the messages for the FG iteration. It replaces the messages in $r_{h}$ as expressed in (13) and (14), and obtains the relative distance messages in variable node $\Delta x_{h}$ and $\Delta y_{h}$ as expressed in (15) to (18). Initial values of $m_{\Delta x_{h} \rightarrow C_{h}}^{2}$ and $m_{\Delta y_{h} \rightarrow C_{h}}^{2}$ are set to 0 , while $\sigma_{\Delta x_{h} \rightarrow C_{h}}^{2}, \sigma_{\Delta y_{h} \rightarrow C_{h}}^{2}$ are set to 1 for the first iteration.

$$
\begin{gathered}
m_{C_{h} \rightarrow r_{h}}=\sqrt{m_{\Delta x_{h} \rightarrow C_{h}}^{2}+m_{\Delta y_{h} \rightarrow C_{h}}^{2}}, \\
\sigma_{C_{h} \rightarrow r_{h}}^{2}=\frac{m_{\Delta x_{h} \rightarrow C_{h}}^{2} \cdot \sigma_{\Delta x_{h} \rightarrow C_{h}}^{2}+m_{\Delta y_{h} \rightarrow C_{h}}^{2} \cdot \sigma_{\Delta y_{h} \rightarrow C_{h}}^{2}}{m_{\Delta x_{h} \rightarrow C_{h}}^{2}+m_{\Delta y_{h} \rightarrow C_{h}}^{2}}, \\
m_{C_{h} \rightarrow \Delta x_{h}}= \pm \sqrt{m_{r_{h} \rightarrow C_{h}}^{2}-m_{\Delta y_{h} \rightarrow C_{h}}^{2}}, \\
m_{C_{h} \rightarrow \Delta y_{h}}= \pm \sqrt{m_{r_{h} \rightarrow C_{h}}^{2}-m_{\Delta x_{h} \rightarrow C_{h}}^{2}}, \\
\sigma_{C_{h} \rightarrow \Delta x_{h}}^{2}=\frac{m_{r_{h} \rightarrow C_{h}}^{2} \cdot \sigma_{r_{h} \rightarrow C_{h}}^{2}+m_{\Delta y_{h} \rightarrow C_{h}}^{2} \cdot \sigma_{\Delta y_{h} \rightarrow C_{h}}^{2}}{m_{r_{h} \rightarrow C_{h}}^{2}-m_{\Delta y_{h} \rightarrow C_{h}}^{2}} \\
\sigma_{C_{h} \rightarrow \Delta y_{h}}^{2}=\frac{m_{r_{h} \rightarrow C_{h}}^{2} \cdot \sigma_{r_{h} \rightarrow C_{h}}^{2}+m_{\Delta x_{h} \rightarrow C_{h}}^{2} \cdot \sigma_{\Delta x_{h} \rightarrow C_{h}}^{2}}{m_{r_{h} \rightarrow C_{h}}^{2}-m_{\Delta x_{h} \rightarrow C_{h}}^{2}}
\end{gathered}
$$

There are three additional constraints to keep stability of the updated message of this node. The constraint (19) is to avoid the value of updated message to diverge, the constraint (20) is to avoid the value of updated messages shrink to zero, while the constraint (21) is to avoid the negative value which resulting the complex number in the following operations.

$$
\begin{aligned}
& \text { if } \quad \begin{aligned}
\sigma_{C \rightarrow r}^{2} & >\max \left(\sigma_{D \rightarrow r}^{2}\right) \\
\sigma_{C \rightarrow r}^{2} & =\max \left(\sigma_{D \rightarrow r}^{2}\right)
\end{aligned} \\
& \text { end, } \\
& \text { if } \quad m_{r \rightarrow C}<\epsilon \\
& m_{r \rightarrow C}=\epsilon
\end{aligned}
$$

$$
\text { if } \quad \begin{aligned}
& m_{r_{h} \rightarrow C_{h}}^{2}-m_{b}^{2}<0 \\
& \\
& m_{b}^{2}=\operatorname{sign}\left(m_{b}\right) \times\left(\operatorname{abs}\left(m_{r_{h} \rightarrow C_{h}}^{2}\right)-\delta\right)
\end{aligned}
$$

end,

where constants $\epsilon$ in (20) and $\delta$ in (21) are ad-hoc parameters which are chosen empirically and $b$ can be either $m_{C_{h} \rightarrow \Delta x_{h}}$ or $m_{C_{h} \rightarrow \Delta y_{h}}$.

Step-7 It is similarly to Step-3, since there are only two nodes directly connected to the relative distance nodes $\Delta x_{i}$ and $\Delta y_{i}$. The means $\left(m_{A_{h} \rightarrow \Delta x_{h}}, m_{B_{h} \rightarrow \Delta y_{h}}\right)$ are set at 0 and variances $\left(\sigma_{A_{h} \rightarrow \Delta x_{h}}^{2}, \sigma_{B_{h} \rightarrow \Delta y_{h}}^{2}\right)$ are set at 1 as the initial value for the first iteration. Step-7 is expressed as

$$
\begin{aligned}
m_{\Delta x_{h} \rightarrow C_{h}} & =m_{A_{h} \rightarrow \Delta x_{h}}, \\
m_{\Delta y_{h} \rightarrow C_{h}} & =m_{B_{h} \rightarrow \Delta y_{h}}, \\
\sigma_{\Delta x_{h} \rightarrow C_{h}}^{2} & =\sigma_{A_{h} \rightarrow \Delta x_{h}}^{2}, \\
\sigma_{\Delta y_{h} \rightarrow C_{h}}^{2} & =\sigma_{B_{h} \rightarrow \Delta y_{h}}^{2}, \\
m_{\Delta x_{h} \rightarrow A_{h}} & =m_{C_{h} \rightarrow \Delta x_{h}} \\
m_{\Delta y_{h} \rightarrow B_{h}} & =m_{C_{h} \rightarrow \Delta y_{h}} \\
\sigma_{\Delta x_{h} \rightarrow A_{h}}^{2} & =\sigma_{C_{h} \rightarrow \Delta x_{h}}^{2} \\
\sigma_{\Delta y_{h} \rightarrow B_{h}}^{2} & =\sigma_{C_{h} \rightarrow \Delta y_{h}}^{2}
\end{aligned}
$$

Step-8 The factor nodes $A_{h}$ and $B_{h}$ convert the messages of the relative distance which are calculated in the variable nodes $x_{i n i t}$ and $y_{\text {init }}$. The operations in these nodes are express as

$$
\begin{gathered}
m_{A_{h} \rightarrow x_{i n i t}}=X_{h}-m_{\Delta x_{h} \rightarrow A_{h}}, \\
m_{B_{h} \rightarrow y_{i n i t}}=Y_{h}-m_{\Delta y_{h} \rightarrow B_{h}}, \\
\sigma_{A_{h} \rightarrow x_{i n i t}}^{2}=\sigma_{\Delta x_{h} \rightarrow A_{h}}^{2}, \\
\sigma_{B_{h} \rightarrow y_{i n i t}}^{2}=\sigma_{\Delta y_{h} \rightarrow B_{h}}^{2}, \\
m_{A_{h} \rightarrow \Delta x_{h}}=X_{h}-m_{x_{i n i t} \rightarrow A_{h}}, \\
m_{B_{h} \rightarrow \Delta y_{h}}=Y_{h}-m_{y_{i n i t} \rightarrow B_{h}}, \\
\sigma_{A_{h} \rightarrow \Delta x_{h}}^{2}=\sigma_{x_{i n i t} \rightarrow A_{h}}^{2}, \\
\sigma_{B_{h} \rightarrow \Delta y_{h}}^{2}=\sigma_{y_{i n i t} \rightarrow B_{h}}^{2} .
\end{gathered}
$$

Step-9 In the iteration process, the variable nodes $x_{\text {init }}$ and $y_{\text {init }}$ sum the messages according to the sum-product algorithm as expressed in (30) and (31) and send the result back to factor nodes $A_{h}$ and $B_{h}$.

$$
\begin{gathered}
\frac{1}{\sigma_{x_{i n i t} \rightarrow A_{h}}^{2}}=\sum_{n=1, n \neq h}^{N} \frac{1}{\sigma_{A_{n} \rightarrow x_{i n i t}}^{2}}, \\
m_{x_{i n i t} \rightarrow A_{h}}=\sigma_{x_{i n i t} \rightarrow A_{h}}^{2} \cdot \sum_{n=1, n \neq h}^{N} \frac{m_{A_{n} \rightarrow x_{i n i t}}}{\sigma_{A_{n} \rightarrow x_{i n i t}}^{2}} .
\end{gathered}
$$

Equations (30) and (31) for the variable node $x_{\text {init }}$ can be applied in the same way as to the variable node $y_{\text {init }}$.

Step-10 Repeat the processes of Step-4 to Step-9 until the iteration converges.

Step-11 When the iteration converges, the variable nodes $x_{\text {init }}$ and $y_{\text {init }}$ sum all incoming messages as

$$
\frac{1}{\sigma_{x_{\text {init }}}^{2}}=\sum_{n=1}^{N} \frac{1}{\sigma_{A_{n} \rightarrow x_{i n i t}}^{2}}
$$




$$
m_{x_{i n i t}}=\sigma_{x_{i n i t}}^{2} \cdot \sum_{n=1}^{N} \frac{m_{A_{n} \rightarrow x_{i n i t}}}{\sigma_{A_{n} \rightarrow x_{i n i t}}^{2}} .
$$

The operations to obtain $m_{y_{\text {init }}}$ are exactly in the same way as (32) and (33).

Then the final values of $\left(m_{x_{i n i t}}, m_{y_{i n i t}}\right)$ represent the rough position estimate of the target obtained by PTDOA-FG. The values of $\left(m_{x_{i n i t}}, m_{y_{i n i t}}\right)$ are used as the initial point of the DRSS-FG technique as well to select the four most suitable monitoring spots having the closest position to the target. The more details of derivation of Step-1 to Step-10, reader may refer to Chapter 4 in [1].

\subsection{DRSS-FG Technique}

By using DRSS-FG, the position of the target $(x, y)$ can be estimated via the relationship between the differential the received signal powers, obtained from the sensors, and the path-loss model estimated from the training data. It should be noticed that the training data, which the RSS samples of monitoring spots are collected during the system off-line, is the result of averaging over long enough duration of measurements to eliminate the shadowing and instantaneous fading. Hence, the RSS samples are assumed in the path-loss model expressed as

$$
P_{h}(d)=20 \log \left(\frac{4 \pi d_{0} f_{c}}{c}\right)+10 n \log \left(\frac{d}{d_{0}}\right),
$$

where $P$ is path-loss exponent in $\mathrm{dB}, d, d_{o}, f_{c}, c$ and $n$ are Eculidean distance from $h$-th sensor to monitoring spot or target in meter, reference distance of exponential model of path-loss in meter, carrier frequency in $\mathrm{Hz}$, velocity of light in $\mathrm{m} / \mathrm{s}$, and coefficient of path-loss exponent, respectively.

For simplicity, in this subsection, the flow of the DRSS-FG run in the fusion center is also briefly described step by step.

Step-1 Factor node $H_{p_{h}}$ provides RSS samples sent by the sensors $\hat{P}_{w_{h}}$, which is originally in units of watt, then converted to logarithmic scale of $\mathrm{dB}, \hat{P}_{h}$.

Step-2 Variable node $N_{p_{h}}$ passes the messages from factor node $H_{p_{h}}$ to factor node $G_{p_{i, j}}$.

Step-3 Factor node $G_{p_{i, j}}$ performs subtracting the logarithmic RSS samples between two sensors to obtain DRSS samples expressed as

$$
\hat{P}_{i, j}=\hat{P}_{i}-\hat{P}_{j},
$$

and then calculates the mean $m_{\hat{P}_{i, j}}$ and the variance $\sigma_{\hat{P}_{i, j}}^{2}$ to be used as the DRSS input to factor node $F_{P_{i, j}}$.

Step-4 Variable node $N_{p_{i, j}}$ directly forwards the messages from the factor node $G_{p_{i, j}}$ to node $F_{P_{i, j}}$.

Step-5 Factor node $F_{P_{i, j}}$ creates linear plane to approximate the position of target utilizing the training data sent from four appropriate monitoring spots, where target is inside of their grid. As detailed presented in [11], the equation for creating the linear plane is expressed as follow

$$
a_{x_{i, j}} \cdot x+a_{y_{i, j}} \cdot y+a_{P_{i, j}} \cdot P_{i, j}=c_{i, j},
$$

where $a_{x_{i, j}}, a_{y_{i, j}}$, and $a_{P_{i, j}}$ are coefficients of the plane equation; $x$ and $y$ denotes the target position; and $c_{i, j}$ is an arbitrary non-zero constant which is set to one in this paper.

From this equation (36), we obtain the the LS solution in matrix form as [11]

$$
\mathbf{a}=\left(\mathbf{B}^{T} \cdot \mathbf{B}\right)^{-1} \cdot \mathbf{B}^{T} \cdot \mathbf{c},
$$

where $\mathbf{a}$ is a vector having its coefficients $a_{x_{i, j}}, a_{y_{i, j}}, a_{P_{i, j}}$, $\mathbf{B}$ is matrix of $x, y$, and $p$, and $\mathbf{c}$ is vector of the non-zero constant which is set 1 in this paper.

Then, the mean $\left(m_{F_{P_{i, j}} \rightarrow x}, m_{F_{P_{i, j}} \rightarrow y}\right)$ and variance $\left(\sigma_{F_{P_{i, j}} \rightarrow x}^{2}, \sigma_{F_{P_{i, j}} \rightarrow y}^{2}\right)$ can be obtained as

$$
\begin{gathered}
m_{F_{P_{i, j}} \rightarrow x}=\alpha_{x_{i, j}}+\beta_{x_{i, j}} \cdot m_{y_{i, j} \rightarrow F_{P_{i, j}}}+\gamma_{x_{i, j}} \cdot m_{\hat{P}_{i, j}}, \\
m_{F_{P_{i, j}} \rightarrow y}=\alpha_{y_{i, j}}+\beta_{y_{i, j}} \cdot m_{x_{i, j} \rightarrow F_{P_{i, j}}}+\gamma_{y_{i, j}} \cdot m_{\hat{P}_{i, j}}, \\
\sigma_{F_{P_{i, j}} \rightarrow x}^{2}=\beta_{x_{i, j}}^{2} \cdot \sigma_{y_{i, j} \rightarrow F_{P_{i, j}}}^{2}+\gamma_{x_{i, j}}^{2} \cdot m_{\hat{P}_{i, j}}, \\
\sigma_{F_{P_{i, j}} \rightarrow y}^{2}=\beta_{y_{i, j}}^{2} \cdot \sigma_{x_{i, j} \rightarrow F_{P_{i, j}}}^{2}+\gamma_{y_{i, j}}^{2} \cdot m_{\hat{P}_{i, j}},
\end{gathered}
$$

where

$$
\begin{aligned}
\alpha_{x_{i, j}} & =c / a_{x_{i, j}}, & \alpha_{y_{i, j}} & =c / a_{y_{i, j}}, \\
\beta_{x_{i, j}} & =-a_{y_{i, j}} / a_{x_{i, j}}, & \beta_{y_{i, j}} & =-a_{x_{i, j}} / a_{y_{i, j}}, \\
\gamma_{x_{i, j}} & =-a_{P_{i, j}} / a_{x_{i, j}}, & \gamma_{y_{i, j}} & =-a_{P_{i, j}} / a_{y_{i, j}} .
\end{aligned}
$$

Step-6 The messages are iteratively exchanged between the factor node $F_{P_{i, j}}$ and variable nodes $x$ and $y$ until they converge to determine the estimated target position $(x, y)$ by performing the sum-product algorithm as in the operations expressed in (30) to (33).

All the notations are summarized in Table 1. For the detail function of each step, except for Step-3, can be found in [11], and more detail explanation regarding DRSS part, particularly the explanation for factor node $G_{p_{i, j}}$ explanation, can be found in [1].

\section{Simulation Results}

This section describes the result of the series computer simulations conducted using Matlab to evaluate the performance of the proposed technique, while the real experiment to verify the proposed technique is left for the next publication because the current real measurement data is not large enough. The simulation area is set in $1,000 \times 1,000 \mathrm{~m}^{2}$, having 3 separated sensors and 1 static unknown target which the position of each trial is randomly chosen as shown in Fig. 2. All sensors $\left(X_{h}, Y_{h}\right)$ are assumed to be synchronized to each other. However, in practice, it may difficult to set perfect synchronism up to $\mu \mathrm{s}$ scale. Therefore, in this paper, we set $100 \mathrm{~m}$ of standard deviation (STD) in the computer simulations for TDOA part, and the SNR value for DRSS part is chosen empirically. The detail of simulation parameter is provided in Table 2.

Since the target is chosen randomly, there are, at least, three possible cases as shown in Fig. (4a), (4c), (4e) indicating the simulation result, which Fig. (4b), (4d), (4f) are the zoomed version of $(4 a),(4 c),(4 e)$, respectively. The first case as shown in Fig. (3a), so called the idealistic case, i.e., when the target takes place in the center of monitoring spot grid, surrounded by four monitoring spots, and the initial point given by PTDOA-FG result placed in the same grid. It should be noticed that four 
TABLE 1: The notations in each node.

\begin{tabular}{|c|c|c|c|}
\hline \multirow{2}{*}{ Messages flow } & \multicolumn{3}{|c|}{ Means, Variances } \\
\hline & Inputs & Outputs & Remarks \\
\hline$E_{i, j} \rightarrow d_{i, j}$ & $\hat{\tau}_{i, j}$ & $\left(m_{E_{i, j} \rightarrow d_{i, j}}, \sigma_{E_{i, j} \rightarrow d_{i, j}}^{2}\right)$ & \multirow{2}{*}{ - Similar to [9] } \\
\hline$d_{i, j} \rightarrow D_{i, j}$ & $\left(m_{E_{i, j} \rightarrow d_{i, j}}, \sigma_{E_{i, j} \rightarrow d_{i, j}}^{2}\right)$ & $\left(m_{d_{i, j} \rightarrow D_{i, j}}, \sigma_{d_{i, j} \rightarrow D_{i, j}}^{2}\right)$ & \\
\hline$D_{i, j} \rightarrow r_{i}$ & $\begin{array}{c}\left(m_{d_{i, j} \rightarrow D_{i, j}}, \sigma_{d_{i, j} \rightarrow D_{i, j}}^{2}\right) \\
\left(m_{r_{i} \rightarrow D_{i, j}}, \sigma_{r_{i} \rightarrow D_{i, j}}^{2}\right)\end{array}$ & $\begin{array}{l}\left(m_{d_{i, j} \rightarrow D_{i, j}}+m_{r_{i} \rightarrow D_{i, j}},\right. \\
\left.\sigma_{d_{i, j} \rightarrow D_{i, j}}^{2}+\sigma_{r_{i} \rightarrow D_{i, j}}^{2}\right)\end{array}$ & \multirow{6}{*}{$\begin{array}{l}\text { - Proposed [1] } \\
\text { - Sensor index is omitted in } r \rightarrow \\
D \text { and } r \rightarrow C \text { for simplicity, while } \\
i, j \in h \text {. The detailed equations are } \\
\text { in (9) - (12) }\end{array}$} \\
\hline$D_{i, j} \rightarrow r_{j}$ & $\left.\begin{array}{c}\left(m_{r_{j} \rightarrow D_{i, j}}, \sigma_{r_{j} \rightarrow D_{i, j}}^{2}\right) \\
\left(m_{d_{i, j} \rightarrow D_{i, j}}, \sigma_{d_{i, j} \rightarrow D_{i, j}}^{2}\right.\end{array}\right)$ & $\begin{array}{l}\left(-m_{d_{i, j \rightarrow D_{i, j}}}+m_{r_{j} \rightarrow D_{i, j}},\right. \\
\left.\sigma_{d_{i, j \rightarrow D_{i, j}}^{2}}^{2}+\sigma_{r_{j} \rightarrow D_{i, j}}^{2}\right)\end{array}$ & \\
\hline $\begin{array}{l}r \rightarrow D \\
r \rightarrow C\end{array}$ & $\begin{array}{c}\left(m_{j}, \sigma_{j}^{2}\right) \\
j \neq i\end{array}$ & $\left(\sigma_{i}^{2} \sum_{j \neq i} \frac{m_{j}}{\sigma_{j}^{2}}, \quad \sigma_{i}^{2}=\frac{1}{\sum_{j \neq i} \frac{1}{\sigma_{j}^{2}}}\right)$ & \\
\hline \multirow[t]{2}{*}{$C_{h} \rightarrow r_{h}$} & $\left(\begin{array}{l}m_{\Delta x_{h} \rightarrow C_{h}}, \sigma_{\Delta x_{h} \rightarrow C_{h}}^{2} \\
m_{\Delta y_{h} \rightarrow C_{h}}, \sigma_{\Delta y_{h} \rightarrow C_{h}}^{2}\end{array}\right)$ & $\begin{array}{l}\left(\sqrt{m_{\Delta x_{h} \rightarrow C_{h}}^{2}+m_{\Delta y_{h} \rightarrow C_{h}}^{2}},\right. \\
\left.\frac{m_{\Delta x_{h} \rightarrow C_{h}}^{2} \sigma_{\Delta x_{h} \rightarrow C_{h}}^{2}+m_{\Delta y_{h} \rightarrow C_{h}}^{2} \sigma_{\Delta y_{h} \rightarrow C_{h}}^{2}}{m_{\Delta x_{h} \rightarrow C_{h}}+m_{\Delta y_{h} \rightarrow C_{h}}^{2}}\right)\end{array}$ & \\
\hline & $\begin{array}{c}\sigma_{C_{h} \rightarrow r_{h}}^{2} \\
\sigma_{r_{r_{h} \rightarrow C_{h}}}^{2}\end{array}$ & Constrain (A) & \\
\hline \multirow{2}{*}{$C_{h} \rightarrow \Delta x_{h}$} & \multirow{2}{*}{$\begin{array}{c}\left(m_{r_{h} \rightarrow C_{h}}, \sigma_{r_{h} \rightarrow C_{h}}^{2}\right) \\
\left(m_{\Delta y_{h} \rightarrow C_{h}}, \sigma_{\Delta y_{h} \rightarrow C_{h}}^{2}\right)\end{array}$} & Constrains (B) and (C) & \\
\hline & & $\begin{array}{l} \pm\left(\sqrt{m_{r_{h} \rightarrow C_{h}}-m_{\Delta y_{h} \rightarrow C_{h}}^{2}},\right. \\
\left.\frac{m_{r_{h} \rightarrow C_{h}}^{2} \sigma_{r_{h} \rightarrow C_{h}}^{2}+m_{\Delta y_{h} \rightarrow C_{h}}^{2} \sigma_{\Delta y_{h} \rightarrow C_{h}}^{2}}{m_{r_{h} \rightarrow C_{h}}^{2}-m_{\Delta y_{h} \rightarrow C_{h}}^{2}}\right)\end{array}$ & \multirow{7}{*}{$\begin{array}{l}\text { - Similar to [9], [11] } \\
\text { - The sign }( \pm) \text { is equal to the } \\
\text { sign of the messages from the } \\
\text { nodes } \Delta y \text { and } \Delta x \text { to the node } \\
C\end{array}$} \\
\hline$C_{h} \rightarrow \Delta y_{h}$ & $\begin{array}{c}\left(m_{r_{h} \rightarrow C_{h}}, \sigma_{r_{h} \rightarrow C_{h}}^{2}\right) \\
\left(m_{\Delta x_{h} \rightarrow C_{h}}, \sigma_{\Delta x_{h} \rightarrow C_{h}}^{2}\right)\end{array}$ & $\begin{array}{l} \pm\left(\sqrt{m_{r_{h} \rightarrow C_{h}}-m_{\Delta x_{h} \rightarrow C_{h}}^{2}},\right. \\
\left.\frac{m_{r_{h} \rightarrow C_{h}}^{2} \sigma_{r_{h} \rightarrow C_{h}}^{2}+m_{\Delta x_{h} \rightarrow C_{h}}^{2} \sigma_{\Delta x_{h} \rightarrow C_{h}}^{2}}{m_{r_{h} \rightarrow C_{h}}^{2}-m_{\Delta x_{h} \rightarrow C_{h}}^{2}}\right)\end{array}$ & \\
\hline $\begin{array}{l}A_{i} \rightarrow \Delta x_{i} \\
A_{i} \rightarrow x\end{array}$ & $\left(m_{i}, \sigma_{i}^{2}\right)$ & $\left(X_{i}-m_{i}, \sigma_{i}^{2}\right)$ & \\
\hline $\begin{array}{l}A_{i} \rightarrow x \\
B_{i} \rightarrow y\end{array}$ & $\left(m_{i}, \sigma_{i}^{2}\right)$ & $\left(Y_{i}-m_{i}, \sigma_{i}^{2}\right)$ & \\
\hline $\begin{array}{l}\Delta x_{i} \rightarrow C_{i} \\
\Delta x_{i} \rightarrow A_{i}\end{array}$ & $\left(m_{i}, \sigma_{i}^{2}\right)$ & - & \\
\hline $\begin{aligned} x_{\text {init }} & \rightarrow A_{i} \\
y_{\text {init }} & \rightarrow B_{i} \\
x & \rightarrow F_{i, j} \\
y & \rightarrow F_{i, j}\end{aligned}$ & $\begin{array}{l}\left(m_{j}, \sigma_{j}^{2}\right) \\
j \neq i\end{array}$ & $\left(\sigma_{i}^{2} \sum_{j \neq i} \frac{m_{j}}{\sigma_{j}^{2}}, \sigma_{i}^{2}=\frac{1}{\sum_{j \neq i} \frac{1}{\sigma_{j}^{2}}}\right)$ & \\
\hline $\begin{array}{l}x_{\text {init }} \text { and } y_{\text {init }} \\
x \text { and } y\end{array}$ & $\left(m_{i}, \sigma_{i}^{2}\right)$ & 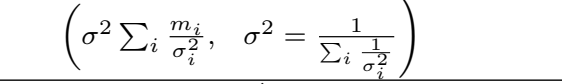 & \\
\hline$H_{p_{h}} \rightarrow N_{h}$ & $\hat{P}_{w, h}$ & $\hat{P}_{h}$ & Similar to [9], [11] \\
\hline$N_{h} \rightarrow G_{i, j}$ & $\hat{P}_{h}$ & $\hat{P}_{h}$ & Proposed \\
\hline$G_{i, j} \rightarrow N_{i, j}$ & $\hat{P}_{i}$ and $\hat{P}_{j}$ & $\left(m_{\hat{P}_{i, j}}, \sigma_{\hat{P}_{i, j}}^{2}\right)$ & Proposed \\
\hline$N_{i, j} \rightarrow F_{i, j}$ & $m_{\hat{P}_{i, j}}, \sigma_{\hat{P}_{i, j}}^{2}$ & $\left(m_{\hat{P}_{i, j}}, \sigma_{\hat{P}_{i, j}}^{2}\right)$ & Similar to [9], [11] \\
\hline$F_{i, j} \rightarrow x$ & $\left.\begin{array}{l}\left(m_{\hat{P}_{i, j}}, \sigma_{\hat{P}_{i, j}}^{2}\right. \\
\left(m_{y_{i, j}}, \sigma_{y_{i, j}}^{2}\right.\end{array}\right)$ & $\left(\frac{c_{i, j}-a_{y_{i, j}} \cdot m_{y_{i, j}}-a_{P_{i, j}} \cdot m_{\hat{P}_{i, j}}}{a_{x_{i, j}}^{2}}\right)$ & Similar to [11] \\
\hline$F_{i, j} \rightarrow y$ & $\left(\begin{array}{l}m_{\hat{P}_{i, j}}, \sigma_{\hat{P}_{i, j}}^{2} \\
m_{x_{i, j}}, \sigma_{x_{i, j}}^{2}\end{array}\right)$ & $\left(\frac{c_{i, j}-a_{x_{i, j}} \cdot m_{x_{i, j}}-a_{P_{i, j}} \cdot m_{\hat{P}_{i, j}}}{a_{y_{i, j}}^{2}}\right)$ & Similar to $[11]$ \\
\hline
\end{tabular}

monitoring spots selected by the proposed technique are exactly the same as the idealistic ones. It is shown in Fig. (4a) that the proposed technique employs the same equation planes formed by the appropriate monitoring spots. Hence, the estimation results of both the proposed technique and the DRSS reference, where DRSS-FG technique with the assumption of having monitoring spots perfectly surrounding the target, meet at the same point as shown in Fig. (4a) (zoomed in Fig. (4b)).

The second case is the occurrence when the target is placed either on the grid line or exactly on the same position of one of the monitoring spots. In this case, there are two possibilities as shown in Figs. (3b) and (3c). It is shown in Fig. (3b) that not all the selected monitoring spots are exactly the same position as the idealistic one. Only two of four monitoring spots are the same position as the idealistic one. However, if the selected monitoring spots are the same as the idealistic ones as shown in Fig. (3c), the result is the same as the first case. The Figs. (4c) and (4d) show the trajectory when the selected monitoring spots are different from the idealistic ones. It can be observed that, even though the final trajectory, indicating the final result, of both proposed technique and DRSS reference are different, but they are still close to the real target.

The third case is the target and the initial point are 
TABLE 2: Simulation Parameters.

\begin{tabular}{|l|c|}
\hline \multicolumn{1}{|c|}{ Parameter } & Value \\
\hline Sensors position $(X, Y)$ & $\begin{array}{c}(100,0),(1100,0), \\
(600,-1000) \mathrm{m}\end{array}$ \\
\hline Target position & arbitrary \\
\hline Simulation area & $1000 \times 1000 \mathrm{~m}^{2}$ \\
\hline Monitoring spot grid & 100 \\
\hline Number of iteration & 100 \\
\hline Number of trial & 1000 \\
\hline Number of sample & $100 \mathrm{~m}$ \\
\hline STD for TDOA & $(0,0) \mathrm{m}$ \\
\hline SNR for DRSS & $1 \mathrm{GHz}$ \\
\hline $\begin{array}{l}\text { Initial point for } \\
\text { DRSS reference }\end{array}$ & $100 \mathrm{~m}$ \\
\hline Frequency carrier $f_{c}$ & 3 \\
\hline Reference distance $d_{0}$ & 3 \\
\hline $\begin{array}{l}\text { Pathloss exponent } \\
\text { for urban area } n\end{array}$ & \\
\hline Epsilon constant $\epsilon$ & $30,40,50) \mathrm{m}$ \\
\hline
\end{tabular}

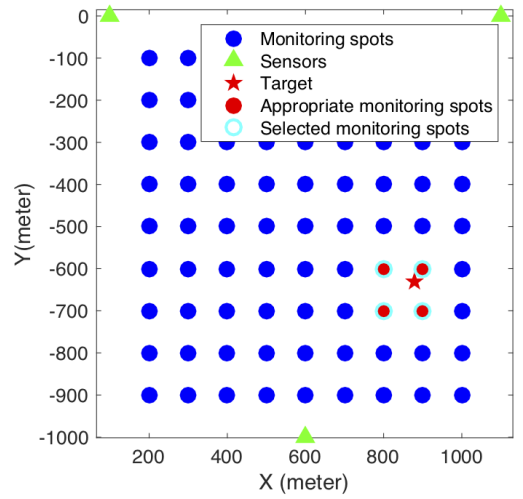

Figure 2: The simulation scenario in $1000 \times 1000 \mathrm{~m}^{2}$ area, $100 \mathrm{~m}$ monitoring spot grid, and the target located arbitrarily.

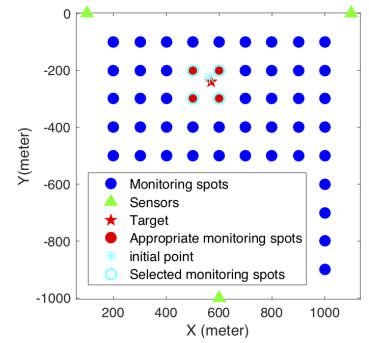

(a)

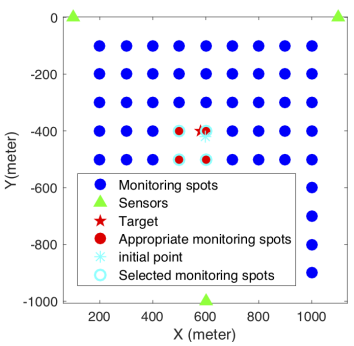

(c)

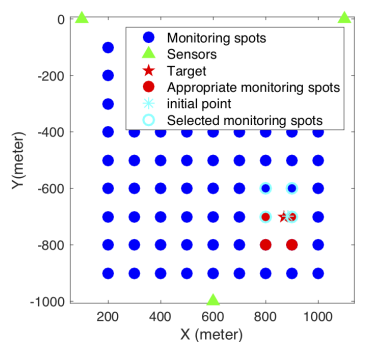

(b)

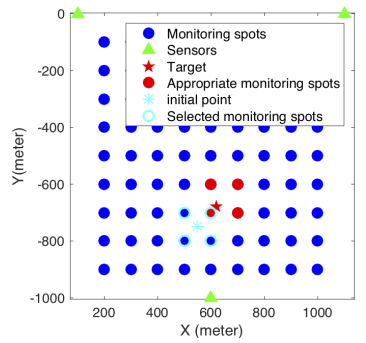

(d)
Figure 3: Trajectory of several cases taking into account the target and the initial point relative to the monitoring spot grid. (3a) is the idealistic case, (3b) and (3c) are the cases where the initial point is on the grid line, (3d) is the case where the initial point in outside of the idealistic grid.

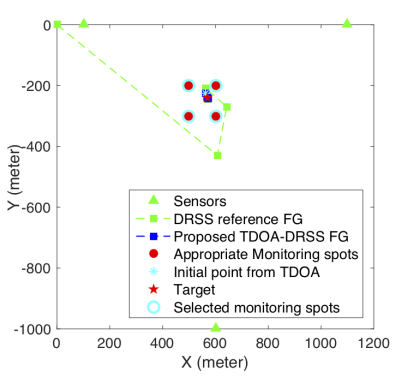

(a)

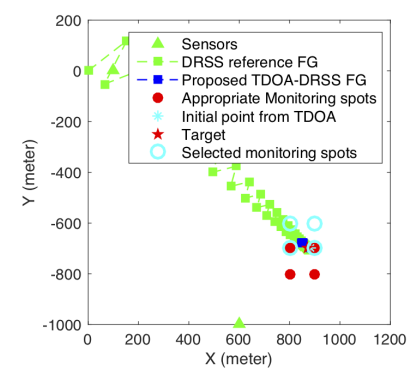

(c)

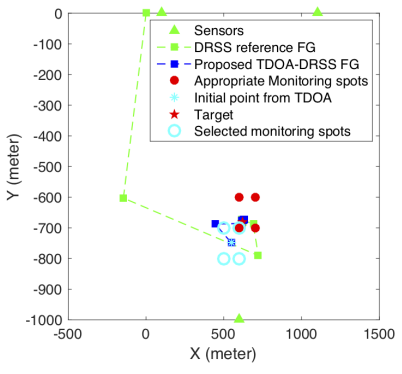

(e)

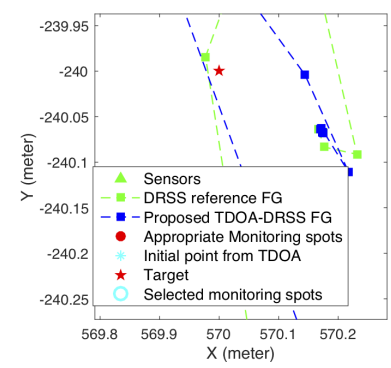

(b)

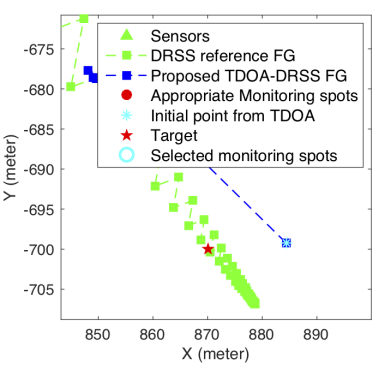

(d)

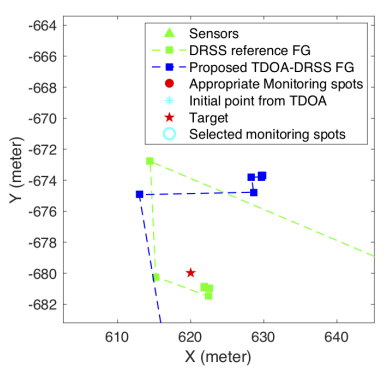

(f)
Figure 4: The trajectory of several cases taking into account the target and the initial point locations toward monitoring spot grid. (4a) trajectory of idealistic case of which zoomed picture in $(4 b),(4 c)$ is trajectory of the grid line case of which zoomed picture in $(4 \mathrm{~d}),(4 \mathrm{e})$ is trajectory of outside area case of which zoomed picture in (4f).

close each other, however, they are in the adjacent grid as shown in Fig. (3d). In this case, DRSS reference with idealistic monitoring spots is providing better estimate than the proposed technique. However, the accuracy gap between the idealistic DRSS-FG and the proposed technique is very small as shown in Fig. (4e), zoomed version in Fig. (4f).

According to the simulations, the idealistic case is the most frequently happen. It can be observed from Fig. 5 that the performance of the proposed technique outperforms the PTDOA-FG alone technique and close to the idealistic DRSS reference performance. The RMSE of the proposed technique is the smallest because the initial point of the proposed is the rough estimation given by the PTDOA-FG, whilst the DRSS reference and PTDOA-FG having the initial point from $(0,0)$. 


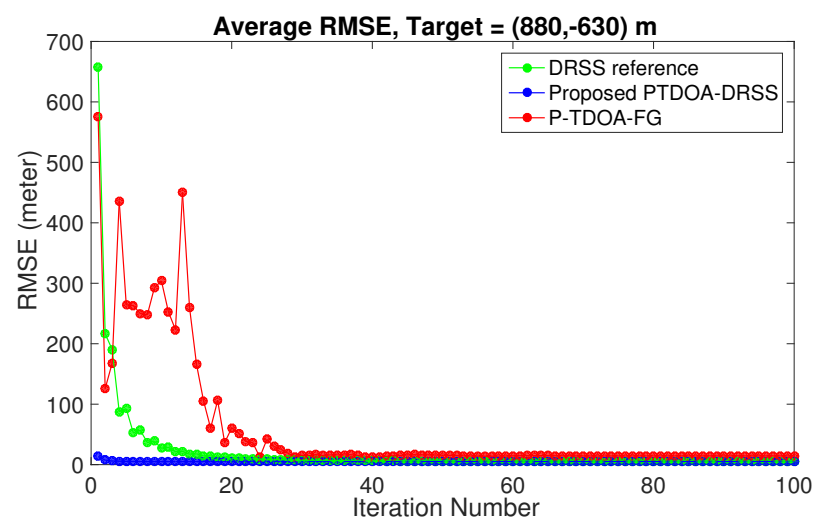

(a)

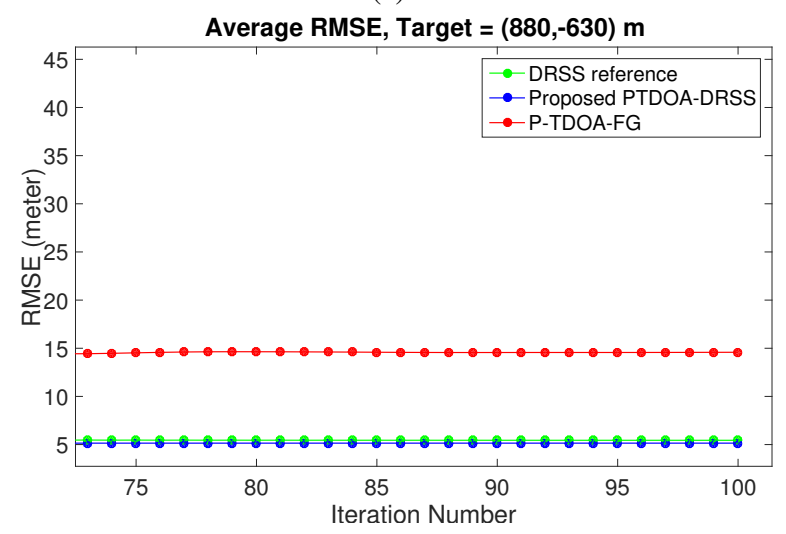

(b)

Figure 5: (5a) The performance of the proposed technique in term of RMSE vs. Iteration number in case the target located in the monitoring spot grid. (5b) is zoomed picture of (5a).

\section{Conclusion}

We have proposed a hybrid PTDOA-DRSS-based FG technique to estimate the location of unknown radio wave emitter, for outdoor environments. With the help of the PTDOA-FG technique to obtain the initial point and to identify the most appropriate monitoring spot positions, the proposed hybrid technique works as good as the idealistic DRSS-FG technique alone. The computer simulation results have confirmed that the proposed technique achieves better accuracy over PTDOA-FG alone because the proposed technique eliminates the problem due to the inaccuracy of timing synchronism among the sensors. Since the DRSS-FG works in the differential domain, it eliminates the necessity of the knowledge of the absolute transmit power of the target. Hence, the proposed hybrid technique requires neither the absolute timing reference information nor the absolute target transmit power information. Verification of the accuracy and the proper operability of the proposed technique in real fields using field-measurement data obtained through measurement campaign is the next step of this work.

\section{Acknowledgment}

This research, in part, supported by Beasiswa Unggulan Kementrian Pendidikan dan Kebudayaan
Indonesia, in part, Kiban (B) No.15H04007, in part, JAIST Core-to-Core Program, and in part, by Koden Electronics Co., Ltd. Authors are very grateful for their support.

\section{References}

[1] M. R. K. Aziz, Factor Graph-Based Geolocation Techniques For Position Detection of Unknown Radio Wave Emitter. A PhD Thesis, Japan Advanced Institute of Science and Technology: JAIST Press, June 2016.

[2] D. Dardari, P. Closas, Salmi, and P. M. Djuric, "Accurate passive location estimation using TOA measurements," IEEE Transactions on Vehicular Technology, vol. 64, no. 4, pp. 1263-1278, April 2015.

[3] C. Gentile, N. Alsindi, R. Raulefs, and C. Teolis, Geolocation Techniques, Principles and Applications. New York: Springer, 2013.

[4] S. N. Karimah, M. R. K. Aziz, and T. Matsumoto, "A hybrid TOA and RSS-based factor graph for wireless geolocation technique," in 2016 IEEE 12th International Colloquium on Signal Processing and its Applications (CSPA2016), March 2016, pp. 140-145.

[5] M. R. K. Aziz, K. Anwar, and T. Matsumoto, "Achieving accurate geo-location detection using joint RSS-DOA factor graph technique," in The International Conference on Telecommunication Systems Services and Applications (TSSA), October 2016.

[6] _ "DRSS-based factor graph geolocation technique for position detection of unknown radio emitter," in Proc. International Workshop on Advanced PHY and MAC Layer Design for 5G Mobile Networks and Internet of Things in conjunction with European WIRELESS, May 2016.

[7] F. R. Kschischang, B. J. Frey, and H.-A. Loeliger, "Factor graphs and the sum-product algorithm," IEEE Trans. on Information Theory, vol. 47, no. 2, pp. 498-519, February 2001.

[8] J.-C. Chen, C.-S. Maa, and J.-T. Chen, "Factor graphs for mobile position location," in Proc. IEEE International Conference on Acoustics, Speech, and Signal Processing (ICASSP) 2003, vol. 2, April 2003, pp. 393-396.

[9] J.-C. Chen, Y.-C. Wang, C.-S. Maa, and J.-T. Chen, "Network side mobile position location using factor graphs," IEEE Trans. on Wireless Comm., vol. 5, no. 10, pp. 2696-2704, October 2006.

[10] - "Mobile position location using factor graphs," IEEE Commun. Lett., vol. 7, pp. 431-433, September 2003.

[11] C.-T. Huang, C.-H. Wu, Y.-N. Lee, and J.-T. Chen, "A novel indoor RSS-based position location algorithm using factor graphs," IEEE Trans. on Wireless Comm., vol. 8, no. 6, pp. 3050-3058, June 2009.

[12] P. Bahl and V. N. Padmanabhan, "Radar: An in-building RF-based user location and tracking system," IEEE INFOCOM 2000, vol. 2, no. , pp. 775-784, March 2000.

[13] M. R. K. Aziz, Y. Lim, and T. Matsumoto, "A new wireless geolocation technique using joint RSS-based voronoi and factor graph," IEEE Computer Society on Conference: Asia Modelling Symposium (AMS) 2015, pp. 131-136, September 2015. 